\title{
Wild ramification of schemes and sheaves
}

\author{
Takeshi Saito *
}

\begin{abstract}
We discuss recent developments on geometric theory of ramification of schemes and sheaves. For invariants of $\ell$-adic cohomology, we present formulas of Riemann-Roch type expressing them in terms of ramification theoretic invariants of sheaves. The latter invariants allow geometric computations involving some new blow-up constructions.
\end{abstract}

Mathematics Subject Classification (2000). Primary 14F20; Secondary 11G25, $11 \mathrm{~S} 15$.

Keywords. Conductor, $\ell$-adic sheaf, wild ramification, Grothendieck-Ogg-Shafarevich formula, Swan class, characteristic class.

\section{Introduction}

For an extension of a number field, the discriminant is an invariant of fundamental importance, in the classical theory of algebraic integers. The celebrated conductor-discriminant formula [40, Chapitre VI Section 3 Corollaire 1] expresses the discriminant as the product of local invariants of ramification, called the conductor. The conductor is defined for a Galois representation, as a measure of the wild ramification. The relation of the conductor of a Galois representation with the level of corresponding modular form plays a crucial role in the quantitative formulation of the Langlands correspondences.

In arithmetic geometry, the conductor showed up in the 60 's in the following scenes among others. For an $\ell$-adic sheaf on a curve over an algebraically closed field of positive characteristic different from $\ell$, the Grothendieck-Ogg-Shafarevich formula [19] computes the Euler number, in geometric terms. The conductor appears in the formula as the local contribution of ramification. The formula is a sheaf theoretic variant of the Riemann-Hurwitz formula, which is a geometric counterpart of the conductor-discriminant formula, with the analogy between the discriminant of a number field and the genus of a curve. Grothendieck raised a question to find a formula of Riemann-Roch type computing the Euler number in higher dimension, which generalizes the GOS formula. Deligne and Laumon deduced a generalization for surfaces using fibration [16] [32], a method different from that taken in this article.

For an elliptic curve over a local field, the Tate-Ogg formula [34] expresses the relation between the discriminant and the conductor of the elliptic curve. In the

*The research is partially supported by JSPS grant-in-aid (B) 18340002 
seminal paper [11], Bloch found a correct generalization to arithmetic schemes in general dimension and proved it for curves. His crucial insight is that the ramification should give rise to an invariant as a 0-cycle class, although the ramification does occur in codimension 1. Kato developed this idea in [28].

In this article, we discuss recent developments on geometric theory of ramification, inspired by the insight of Bloch. Some of related results were already discussed 20 years ago in Kyoto by Kato [27, Section 4]. We will not touch on arithmetic applications of ramifications, including canonical subgroups of abelian varieties [3], [42], explicit computation of local Fourier transform [8], finite flat group schemes [21] etc., although they should not be ignored. We will not discuss either the $p$-adic approach using $p$-adic $\mathcal{D}$-modules [10], see e.g. [9], [33].

The article consists of two parts. In the first part, we introduce an invariant, called the Swan class, as a measure of the wild ramification of a covering of schemes or a sheaf. We present formulas of Riemann-Roch type computing the Euler number or the conductor of cohomology of an $\ell$-adic sheaf in terms of the Swan class, as generalizations of the GOS formula and Bloch's formula. In the geometric case, the characteristic class of an $\ell$-adic sheaf is defined as a cohomology class and is shown to equal the cycle class of the Swan class. This gives a refinement of the generalized GOS formula.

In the second part, we discuss a new geometric method to study the wild ramification, blowing-up at the ramification locus in the diagonal. A traditional approach in the study of ramification of a sheaf, taken in the first part of the article, is to kill the ramification by taking a ramified covering. The new approach replaces ramified coverings by blowing-ups. It grew out of the definition of the upper numbering filtration of ramification groups of the absolute Galois group of a local field with not necessarily perfect residue field. By globalizing the construction, we have a geometric method to study the ramification of a sheaf along the boundary.

At the end of the article, we introduce the characteristic cycle of an $\ell$-adic sheaf satisfying a certain condition and show that it computes the characteristic class and hence the Euler number. An analogy of the wild ramification of $\ell$-adic sheaves with the irregularities of $\mathcal{D}$-modules has been observed by many mathematicians e.g [15], [28]. The author expects that the new geometric approach shed more light on it.

The author would like to thank his coauthors Kazuya Kato and Ahmed Abbes for long time and fruitful collaborations. Large parts of Sections 1.3 and 2.2 are based on papers in preparation coauthored with them, respectively. It should be evident from the article that a considerable part of the contents is due to them.

\section{1. $\ell$-adic Riemann-Roch formulas}

In Sections 1.1 and 1.2, we consider the geometric case where the base field is a perfect field of positive characteristic. We introduce in Section 1.1 the Swan class of an $\ell$-adic sheaf and state a formula for the Euler number, as a generalization of the Grothendieck-Ogg-Shafarevich formula. We define the characteristic class 
in Section 1.2 as a refinement of the Euler number and gives a relation with the Swan class. In Section 1.3, we consider the arithmetic case where the base field is a $p$-adic field with perfect residue field and formulate results analogous to those in Section 1.1.

1.1. Euler numbers. Let $k$ be a perfect field and $U$ be a smooth separated scheme of finite type of dimension $d$ over $k$. For a separated scheme $X$ of finite type over $k$, the Chow group $C H_{0}(X)$ denotes the group of 0 -cycles modulo rational equivalence. We define

$$
C H_{0}(\partial U)=\varliminf_{\lim } C H_{0}(X \backslash U), \quad C H_{0}(\partial V)_{\mathbb{Q}}=\varliminf_{\lim }\left(C H_{0}(X \backslash U) \otimes_{\mathbb{Z}} \mathbb{Q}\right)
$$

to be the projective limits with respect to proper schemes $X$ containing $U$ as a dense open subscheme and proper push-forward. The degree maps $C H_{0}(X \backslash U) \rightarrow$ $C H_{0}($ Spec $k)=\mathbb{Z}$ induce $\operatorname{deg}_{k}: C H_{0}(\partial U) \rightarrow \mathbb{Z}$.

For a finite etale Galois covering $V \rightarrow U$ of Galois group $G$, we define the Swan character class

$$
s_{V / U}(\sigma) \in C H_{0}(\partial V)_{\mathbb{Q}}
$$

for $\sigma \in G$. We refer to [31, Definition 4.1] for the definition in the general case that requires alteration [14], causing the denominator. Here we only give a definition of the image in $\mathrm{CH}_{0}(Y \backslash V)$, for a smooth compactification $Y$ of $V$ satisfying certain good properties.

Assume $Y$ is a proper smooth scheme containing $V$ as the complement of a divisor $D$ with simple normal crossings. Let $D_{1}, \ldots, D_{n}$ be the irreducible components of $D$ and let $\left(Y \times_{k} Y\right)^{\prime} \rightarrow Y \times_{k} Y$ be the blow-up at $D_{i} \times_{k} D_{i}$ for every $i=1, \ldots, n$. Namely the blow-up by the product of the ideal sheaves $\mathcal{I}_{D_{i} \times_{k} D_{i}} \subset \mathcal{O}_{Y \times_{k} Y}$. We call the complement $Y *_{k} Y \subset\left(Y \times_{k} Y\right)^{\prime}$ of the proper transform of $\left(D \times_{k} Y\right) \cup\left(Y \times_{k} D\right)$ the $\log$ product. The diagonal map $\delta: Y \rightarrow Y \times_{k} Y$ is uniquely lifted to a closed immersion $\tilde{\delta}: Y \rightarrow Y *_{k} Y$ called the $\log$ diagonal. The log products and the log diagonal seem to have been first introduced by Faltings [20] and by Pink [36] apparantly independently. For an explicit local description, see Example 2.2 in Section 2.2. For more intrinsic definition in the language of log geometry, we refer to [30, Section 4]. We introduce the $\log$ product in order to focus on the wild ramification. A heuristic reason for this is that a tamely ramified covering can be regarded as an unramified covering in log geometry.

Let $\sigma \in G$ be an element different from the identity and let $\Gamma$ be a closed subscheme of $Y *_{k} Y$ of dimension $d=\operatorname{dim} Y$ such that the intersection $\Gamma \cap\left(V \times_{k} V\right)$ is equal to the graph $\Gamma_{\sigma}$ of $\sigma$. By the assumption that $V$ is etale over $U$, the intersection $\Gamma_{\sigma} \cap \Delta_{V}$ with the diagonal $\Delta_{V}=\delta(V) \subset V \times_{k} V$ is empty. Hence the intersection product $\left(\Gamma, \Delta_{Y}^{\log }\right)_{Y *_{k} Y}$ with the log diagonal $\Delta_{Y}^{\log }=\tilde{\delta}(Y) \subset Y *_{k} Y$ is defined in $C H_{0}(Y \backslash V)$. The intersection product $\left(\Gamma, \Delta_{Y}^{\log }\right)_{Y *_{k} Y}$ is shown to be independent of the choice of $\Gamma$ under the assumption that $V \rightarrow U$ is extended to a map $Y \rightarrow X$ to a proper scheme $X$ over $k$ containing $U$ as the complement of a Cartier divisor $B$ and that the image of $\Gamma$ in the $\log$ product $X *_{k} X$ defined with respect to $B$ is contained in the $\log$ diagonal $\Delta_{X}^{\log }$. 
The Swan chararacter class $s_{V / U}(\sigma) \in C H_{0}(Y \backslash V)$ for $\sigma \neq 1$ is defined by

$$
s_{V / U}(\sigma)=-\left(\Gamma, \Delta_{Y}^{\log }\right)_{Y *_{k} Y} .
$$

For $\sigma=1$, it is defined by requiring $\sum_{\sigma \in G} s_{V / U}(\sigma)=0$. For $\sigma \neq 1$, we have

$$
\sum_{q=0}^{2 \operatorname{dim} V}(-1)^{q} \operatorname{Tr}\left(\sigma^{*}: H_{c}^{q}\left(V_{\bar{k}}, \mathbb{Q}_{\ell}\right)\right)=-\operatorname{deg}_{k} s_{V / U}(\sigma)
$$

by a Lefschetz trace formula for open varieties [31, Theorem 2.3.4] for a prime number $\ell$ different from the characteristic of $k$.

Example 1.1. Assume that $V$ is a curve. Then, $Y$ is unique and we have $C H_{0}(\partial V)=C H_{0}(Y \backslash V)=\bigoplus_{y \in Y \backslash V} \mathbb{Z}$. For $\sigma \neq 1, \in G$, we have

$$
s_{V / U}(\sigma)=-\sum_{y \in\{y \in Y \mid \sigma(y)=y\}} \text { length } \mathcal{O}_{y} /\left(\frac{\sigma(a)}{a}-1 ; a \in \mathcal{O}_{y}, \neq 0\right) \cdot[y] .
$$

Let $\ell$ be a prime number different from $p=\operatorname{char} k>0$. We consider a smooth $\ell$-adic sheaf $\mathcal{F}$ on $U$ and define the Swan class $\mathrm{Sw}_{U} \mathcal{F} \in C H_{0}(\partial U)_{\mathbb{Q}\left(\zeta_{p} \infty\right)}$. We refer to [31, Definition 4.2.2] for the definition in the general case that requires reduction modulo $\ell$ and Brauer traces [22]. Here we only give a definition assuming that there exists a finite etale Galois covering $f: V \rightarrow U$ trivializing $\mathcal{F}$. Let $G$ denote the Galois group $\operatorname{Gal}(V / U)$ and $M$ be the representation of $G$ corresponding to $\mathcal{F}$. Then, the Swan class is defined by

$$
\mathrm{Sw}_{U} \mathcal{F}=\frac{1}{|G|} \sum_{\sigma \in G} f_{*} s_{V / U}(\sigma) \cdot \operatorname{Tr}(\sigma: M) .
$$

By the equality (3), this is an immediate generalization of the classical definition of the Swan conductor [41, Partie III], see also Example 1.3 in Section 1.3. For the Swan class, we expect that the Hasse-Arf theorem [40, Chapitre VI Section 2 Théorème 1] can be generalized as follows:

Conjecture 1.1. The Swan class $\mathrm{Sw}_{U} \mathcal{F} \in C H_{0}(\partial U)_{\mathbb{Q}\left(\zeta_{p} \infty\right)}$ is in the image of $C H_{0}(\partial U)$.

Conjecture 1.1 implies a conjecture of Serre on the integrality of the Artin character for an isolated fixed point [39] in the geometric case. By the standard argument using Brauer induction, it is reduced to the rank 1 case. By computing the Swan class in the rank 1 case using Theorem 2.12, Conjecture 1.1 is proved in [31, Corollary 5.1.7] for $U$ of dimension 2. The conjecture of Serre for surfaces is proved earlier in [29].

For a smooth $\ell$-adic sheaf $\mathcal{F}$ on $U$, the Euler number $\chi_{c}\left(U_{\bar{k}}, \mathcal{F}\right)$ is defined as the alternating sum $\sum_{q=0}^{2 \operatorname{dim} U}(-1)^{q} \operatorname{dim} H_{c}^{q}\left(U_{\bar{k}}, \mathcal{F}\right)$. The Lefschetz trace formula for open varieties (2) implies the following generalization of the Grothendieck-OggShafarevich formula: 
Theorem 1.2 ([31, Theorem 4.2.9]). Let $U$ be a separated smooth scheme of finite type over $k$. For a smooth $\ell$-adic sheaf $\mathcal{F}$ on $U$, we have

$$
\chi_{c}\left(U_{\bar{k}}, \mathcal{F}\right)=\operatorname{rank} \mathcal{F} \cdot \chi_{c}\left(U_{\bar{k}}, \mathbb{Q}_{\ell}\right)-\operatorname{deg}_{k} \operatorname{Sw}_{U} \mathcal{F} .
$$

1.2. Characteristic classes. We recall from [6, Definition 2.1.1] the definition of the characteristic class of an $\ell$-adic sheaf on a separated scheme of finite type over a field $k$ of characteristic different from $\ell$. Although it is not stated explicitly, essential ingredients in the definition are contained in [18], see also [24, Section 9.1].

Let $X$ be a separated scheme of finite type over a field $k$. As a coefficient ring $\Lambda$, we consider a ring finite over $\mathbb{Z} / \ell^{n} \mathbb{Z}, \mathbb{Z}_{\ell}$ or $\mathbb{Q}_{\ell}$ for a prime number $\ell \neq$ char $k$. Let $a: X \rightarrow$ Spec $k$ denote the structure map and $K_{X}=R a ! \Lambda$ denote the dualizing complex. If $X$ is smooth of dimension $d$ over $k$, we have $K_{X}=\Lambda(d)[2 d]$.

Let $\mathcal{F}$ be a constructible sheaf of flat $\Lambda$-modules on $X$ and consider the object

$$
\mathcal{H}=R \mathcal{H o m}\left(\operatorname{pr}_{2}^{*} \mathcal{F}, R \operatorname{pr}_{1}^{!} \mathcal{F}\right)
$$

of the derived category $D_{\mathrm{ctf}}\left(X \times_{k} X, \Lambda\right)$ of constructible sheaves of $\Lambda$-modules of finite tor-dimension on the product $X \times_{k} X$. If $X$ is smooth of dimension $d$ over $k$ and if $\mathcal{F}$ is smooth, we have a canonical isomorphism $\mathcal{H} \rightarrow \mathcal{H o m}\left(\operatorname{pr}_{2}^{*} \mathcal{F}, \operatorname{pr}_{1}^{*} \mathcal{F}\right)(d)[2 d]$.

A canonical isomorphism

$$
\operatorname{End}(\mathcal{F}) \rightarrow H_{X}^{0}\left(X \times_{k} X, \mathcal{H}\right)
$$

is defined in [18]. Hence, we may regard the identity $\mathrm{id}_{\mathcal{F}}$ as a cohomology class $\operatorname{id}_{\mathcal{F}} \in H_{X}^{0}\left(X \times{ }_{k} X, \mathcal{H}\right)$ supported on the diagonal $X \subset X \times{ }_{k} X$. Let $\delta: X \rightarrow X \times{ }_{k} X$ be the diagonal map. Further in [18], a canonical map $\delta^{*} \mathcal{H} \rightarrow K_{X}$ is defined as the trace map. The characteristic class

$$
C(\mathcal{F}) \in H^{0}\left(X, K_{X}\right)
$$

is defined as the image of the pull-back $\delta^{*} \operatorname{id}_{\mathcal{F}} \in H^{0}\left(X, \delta^{*} \mathcal{H}\right)$ by the induced map $H^{0}\left(X, \delta^{*} \mathcal{H}\right) \rightarrow H^{0}\left(X, K_{X}\right)$. If $X$ is smooth and if $\mathcal{F}$ is smooth, we have $C(\mathcal{F})=\operatorname{rank} \mathcal{F} \cdot(X, X)_{X \times_{k} X}$ where $(X, X)_{X \times_{k} X}$ denotes the self-intersection in the product $X \times_{k} X$. The Lefschetz trace formula [18] asserts that, if $X$ is proper, the trace map $H^{0}\left(X, K_{X}\right) \rightarrow \Lambda$ sends the characteristic class $C(\mathcal{F})$ to the Euler number $\chi\left(X_{\bar{k}}, \mathcal{F}\right)$. In other words, the characteristic class is a geometric refinement of the Euler number.

By a standard devissage, the computation of the characteristic classes is reduced to that of the difference $C\left(j ! \mathcal{F}_{U}\right)-$ rank $\mathcal{F}_{U} \cdot C(j ! \Lambda)$ where $j: U \rightarrow X$ is the immersion of a dense open subscheme $U \subset X$ smooth over $k$ and $\mathcal{F}_{U}$ is a smooth sheaf of flat $\Lambda$-modules on $U$. Under a certain mild technical assumption on $\mathcal{F}$, the difference is computed by the Swan class as follows.

Theorem 1.3 ([6, Theorem 3.3.1]). Let $U$ be a smooth dense open subscheme of a separated scheme $X$ of finite type over $k$. Let $\Lambda$ be a finite extension of $\mathbb{Q}_{\ell}$ and $\mathcal{F}_{U}$ 
be a smooth $\Lambda$-sheaf on $U$. Assume that there exists a finite etale covering $V \rightarrow U$ such that the pull-back $\mathcal{F}_{V}$ is of Kummer type with respect to the normalization $Y$ of $X$ in $V$.

Then, we have

$$
C\left(j_{!} \mathcal{F}_{U}\right)-\operatorname{rank} \mathcal{F}_{U} \cdot C(j ! \Lambda)=-\left[\mathrm{Sw}_{U} \mathcal{F}_{U}\right]
$$

in $H^{0}\left(X, K_{X}\right)$, where [ ] denotes the cycle class.

We refer to [6, Definition 3.1.1] for the definition of being of Kummer type. We only remark here that the purity theorem of Zariski-Nagata and Abhyankar's lemma [37] imply that the assumption on $\mathcal{F}$ is satisfied if we admit a strong form of resolution of singularities for $Y$. One can also deduce Theorem 1.2 unconditionally from Theorem 1.3.

Problem 1. Find a definition of the characteristic class of an $\ell$-adic sheaf on a separated scheme of finite type over a complete discrete valuation ring with perfect residue field and prove a relation similar to Theorem 1.3 with the Swan class defined in Section 1.3.

1.3. Conductor formula. Let $K$ be a complete discrete valuation field with perfect residue field $F=\mathcal{O}_{K} / \mathfrak{m}_{K}$. For simplicity, we will assume that the characteristic of $K$ is 0 . We consider constructions and formulas for schemes over $K$ analogous to those in Section 1.1. For a scheme $X$ of finite type over $S=\operatorname{Spec} \mathcal{O}_{K}$, let $G(X)$ denote the Grothendieck group of coherent $\mathcal{O}_{X}$-modules and $F_{\bullet}$ be the increasing filtration of $G(X)$ defined by the dimension of support.

Let $U$ be a smooth separated scheme of finite type of dimension $d-1$ over $K$. We define

$$
F_{0} G\left(\partial_{F} U\right)=\lim _{\longleftarrow} F_{0} G\left(X_{F}\right), \quad F_{0} G\left(\partial_{F} U\right)_{\mathbb{Q}}=\varliminf_{\longleftarrow}\left(F_{0} G\left(X_{F}\right) \otimes_{\mathbb{Z}} \mathbb{Q}\right)
$$

to be the projective limits with respect to schemes $X$ proper over $S$ containing $U$ as a dense open subscheme and proper push-forward. For a morphism $f: U \rightarrow V$ of separated smooth schemes of finite type over $K$, the push-forward maps define a map $f_{!}: F_{0} G\left(\partial_{F} U\right) \rightarrow F_{0} G\left(\partial_{F} V\right)$. In particular, for $V=$ Spec $K$, the degree $\operatorname{map} \operatorname{deg}_{F}: F_{0} G\left(\partial_{F} U\right) \rightarrow \mathbb{Z}$ is defined.

For a finite etale Galois covering $V \rightarrow U$ of Galois group $G$, we define the Swan character class

$$
s_{V / U}(\sigma) \in F_{0} G\left(\partial_{F} V\right)_{\mathbb{Q}}
$$

for $\sigma \in G$. Here we only sketch the definition of the image in $F_{0} G\left(Y_{F}\right)$, for a smooth compactification $Y$ of $V$ satisfying certain good properties similarly as in Section 1.1.

Assume $Y$ is a proper regular flat scheme over $S$ containing $V$ as the complement of a divisor $D$ with simple normal crossings. Then, we define the log product $Y *_{S} Y$ similarly to $Y *_{k} Y$. The diagonal map $\delta: Y \rightarrow Y \times_{S} Y$ is uniquely lifted to a closed immersion $\tilde{\delta}: Y \rightarrow Y *_{S} Y$ called the log diagonal. 
Let $\sigma \in G$ be an element and let $\Gamma$ be a closed subscheme of $Y *_{S} Y$ of dimension $d=\operatorname{dim} Y$ such that the intersection $\Gamma \cap\left(V \times{ }_{S} V\right)$ is equal to the graph $\Gamma_{\sigma}$ of $\sigma$. The localized $K$-theoretic intersection product $\left(\left(\Gamma, \Delta_{Y}^{\log }\right)\right)_{Y *_{S} Y} \in F_{0} G\left(Y_{F}\right)$ is then defined by

$$
\left(\left(\Gamma, \Delta_{Y}^{\log }\right)\right)_{Y{ }_{S} Y}=(-1)^{q}\left(\left[\mathcal{T}_{\text {or }_{q}}^{\mathcal{O}_{Y *{ }_{S} Y}}\left(\mathcal{O}_{\Gamma}, \mathcal{O}_{\Delta_{Y}^{\log }}\right)\right]-\left[\mathcal{T} \operatorname{or}_{q+1}^{\mathcal{O}_{Y * S}{ }^{Y}}\left(\mathcal{O}_{\Gamma}, \mathcal{O}_{\Delta_{Y}^{\log }}\right)\right]\right)
$$

for $q \geq d=\operatorname{dim} Y$ [30, Section 3]. It is a non-trivial fact that the right hand side is independent of the choice of $\Gamma$ or $q \geq d$, under an assumption similar to the corresponding one in Section 1.1. We write $\left(\left(\Gamma_{\sigma}, \Delta_{V}\right)\right)$ for $\left(\left(\Gamma, \Delta_{Y}^{\log }\right)\right)_{Y *_{S} Y}$.

The Swan chararacter class $s_{V / U}(\sigma) \in F_{0} G\left(Y_{F}\right)$ for $\sigma \neq 1$ is defined by

$$
s_{V / U}(\sigma)=-\left(\left(\Gamma_{\sigma}, \Delta_{V}\right)\right)
$$

For $\sigma=1$, it is defined by requiring $\sum_{\sigma \in G} s_{V / U}(\sigma)=0$.

Example 1.2. Assume that $V=\operatorname{Spec} L$ for a totally ramified extension of $K$. Then, $Y=\operatorname{Spec} \mathcal{O}_{L}$ is unique and we have $F_{0} G\left(\partial_{F} V\right)=F_{0} G(\operatorname{Spec} F)=\mathbb{Z}$. The $\log$ product $Y *_{S} Y$ is $\operatorname{Spec} \mathcal{O}_{L} \otimes \mathcal{O}_{K} \mathcal{O}_{L}\left[U^{ \pm 1}\right] /(1 \otimes t-t \otimes 1 \cdot U)$ for a prime element $t$ of $L$, by definition. The minimal polynomial $f \in \mathcal{O}_{K}[T]$ of $t$ is an Eisenstein polynomial. We have $Y *_{S} Y=\operatorname{Spec} \mathcal{O}_{L}\left[U^{ \pm 1}\right] /(f(t U))$.

Assume $L$ is a Galois extension and let $\sigma$ be an element of $G=\operatorname{Gal}(L / K)$. We define $g_{\sigma} \in \mathcal{O}_{L}[U]$ by $f(t U)=g_{\sigma} \cdot(U-\sigma(t) / t)$ and put $A=\mathcal{O}_{L}\left[U^{ \pm 1}\right] /(f(t U))$, $\mathcal{O}_{\sigma}=A /(U-\sigma(t) / t)$. Then we have a periodic free resolution

$$
\cdots \longrightarrow A \stackrel{\times(U-\sigma(t) / t)}{\longrightarrow} A \stackrel{\times g_{\sigma}}{\longrightarrow} A \stackrel{\times(U-\sigma(t) / t)}{\longrightarrow} A \longrightarrow \mathcal{O}_{\sigma} \rightarrow 0
$$

Hence, for $\sigma \neq 1$, we have

$$
\mathcal{T}_{o r}^{A}\left(\mathcal{O}_{\sigma}, \mathcal{O}_{1}\right)= \begin{cases}\mathcal{O}_{L} /(\sigma(t) / t-1) & \text { if } q \text { is even } \\ 0 & \text { if } q \text { is odd }\end{cases}
$$

Consequently, we have

$$
s_{V / U}(\sigma)=-\operatorname{ord}_{L}\left(\frac{\sigma(t)}{t}-1\right) .
$$

For a smooth $\ell$-adic sheaf $\mathcal{F}$ on $U$, the Swan class $\mathrm{Sw}_{U} \mathcal{F} \in F_{0} G\left(\partial_{F} U\right)_{\mathbb{Q}\left(\zeta_{p} \infty\right)}$ is defined. Under the same simplifying assumptions, the Swan class is defined by the same formula (4) as in the geometric case. By the equality (10), this is an immediate generalization of the classical definition of the Swan conductor as follows.

Example 1.3. We consider $\mathcal{F}$ on $U=\operatorname{Spec} K$ corresponding to an $\ell$-adic repreresentation $M$ of the absolute Galois group $G_{K}=\operatorname{Gal}(\bar{K} / K)$ factoring through 
a finite quotient $G=\operatorname{Gal}(L / K)$. Then, the Swan class $\operatorname{Sw}_{U} \mathcal{F}$ is nothing but the Swan conductor $\mathrm{Sw}_{K} M \in \mathbb{Z}=C H_{0}\left(\partial_{F} U\right)=C H_{0}(\operatorname{Spec} F)$ defined by

$$
\mathrm{Sw}_{K} M=\frac{1}{|G|} \sum_{\sigma \in G} s_{L / K}(\sigma) \cdot \operatorname{Tr}(\sigma: M) .
$$

For the Swan class defined above, we also expect that the Hasse-Arf theorem can be generalized as in Conjecture 1.1. As in the geometric case, it is proved for a curve $U$ over $K$. A proof of the conjecture of Serre [39] in the corresponding case is announced in [27], see also [1], [2].

Let $U_{1} \subset U$ be a regular closed subscheme and $i: U_{1} \rightarrow U$ and $j: U_{0}=U \backslash U_{1} \rightarrow$ $U$ denote the immersions. Then, we have an excision formula

$$
\mathrm{Sw}_{U} \mathcal{F}=\left.j ! \mathrm{Sw}_{U_{0}} \mathcal{F}\right|_{U_{0}}+\left.i_{!} \mathrm{Sw}_{U_{1}} \mathcal{F}\right|_{U_{1}} \text {. }
$$

This enables us to extend the definition of the Swan classes to constructible sheaves. For a smooth sheaf $\mathcal{F}$ on $U$, we define a variant of the Swan class by

$$
\overline{\mathrm{Sw}}_{U} \mathcal{F}=-\operatorname{rank} \mathcal{F} \cdot\left(\left(\Delta_{U}, \Delta_{U}\right)\right)+\mathrm{Sw}_{U} \mathcal{F} .
$$

This is also extended to constructible sheaves by the excision formula.

For the variant, we have the following formula of Riemann-Roch type:

Theorem 1.4. Assume $K$ is of characteristic 0 . For a morphism $f: U \rightarrow V$ of separated schemes of finite type over $K$ and for a constructible $\ell$-adic sheaf $\mathcal{F}$ on $U$, we have

$$
\overline{\mathrm{Sw}}_{V} R f_{!} \mathcal{F}=f_{!} \overline{\mathrm{Sw}}_{U} \mathcal{F} .
$$

The outline of the proof is as follows. By standard devissage using the excision formula, it is reduced to the relative curve case. Then, we take an alteration and apply a logarithmic Lefschetz trace formula for an open variety over a local field generalizing [30, Theorem 6.5.1], to conclude the proof.

In the case where $V=\operatorname{Spec} K$, Theorem 1.4 gives a conductor formula. For a smooth $\ell$-adic sheaf $\mathcal{F}$ on a separated scheme $U$ of finite type over $K$, let $\mathrm{Sw}_{K} H_{c}^{*}\left(U_{\bar{K}}, \mathcal{F}\right)$ denote the alternating sum $\sum_{q=0}^{2 \operatorname{dim} U}(-1)^{q} \mathrm{Sw}_{K} H_{c}^{q}\left(U_{\bar{K}}, \mathcal{F}\right)$ of the Swan conductor.

Corollary. Let $U$ be a separated smooth scheme of finite type of dimension $d-1$ over $K$.

1. For a smooth $\ell$-adic sheaf $\mathcal{F}$ on $U$, we have

$$
\mathrm{Sw}_{K} H_{c}^{*}\left(U_{\bar{K}}, \mathcal{F}\right)=\operatorname{rank} \mathcal{F} \cdot \operatorname{Sw}_{K} H_{c}^{*}\left(U_{\bar{K}}, \mathbb{Q}_{\ell}\right)+\operatorname{deg}_{F} \operatorname{Sw}_{U} \mathcal{F} .
$$

2. ([30, Theorem 6.2.3]) Assume $U$ is proper over $K$ and let $X$ be a proper regular flat scheme such that $U=X_{K}$. Assume that the reduced closed fiber $X_{F, \text { red }}$ is a divisor with simple normal crossings. Then, we have

$$
\chi_{c}\left(X_{\bar{K}}, \mathbb{Q}_{\ell}\right)-\chi_{c}\left(X_{\bar{F}}, \mathbb{Q}_{\ell}\right)+\operatorname{Sw}_{K} H_{c}^{*}\left(X_{\bar{K}}, \mathbb{Q}_{\ell}\right)=(-1)^{d-1} \operatorname{deg}_{F} c_{d}\left(\Omega_{X / S}^{1}\right),
$$

where $c_{d}\left(\Omega_{X / S}^{1}\right) \in C H_{0}\left(X_{F}\right)$ denotes the localized Chern class. 
The formula (15) is conjectured by Bloch in [11] and proved there for curves. For surfaces $\operatorname{dim} U=2$, we can remove the assumption on the reduced closed fiber $X_{F, \text { red }}$, since the strong resolution of singularity is now obtained by blow-up in dimension 2 [23]. In a geometric case, a formula analogous to (14) is obtained by using a localized refinement of the characteristic class in [43], assuming resolution of singularities.

\section{Geometric ramification theory}

We recall the geometric definition of the filtration by ramification groups of Galois groups of local fields in Section 2.1. We globalize it in Section 2.2 and study the ramification of a Galois covering of a smooth scheme over a perfect field of characteristic $p>0$ along the boundary. We compute in Section 2.3 the characteristic class using the construction in Section 2.2 and introduce the characteristic cycle of an $\ell$-adic sheaf that enables one to compute the Euler number.

2.1. Ramification groups of a local field. Let $K$ be a complete discrete valuation field with not necessarily perfect residue field $F=\mathcal{O}_{K} / \mathfrak{m}_{K}$. For a finite Galois extension $L$ over $K$, the Galois group $G=\operatorname{Gal}(L / K)$ has two decreasing filtrations, the lower numbering filtration $\left(G_{i}\right)_{i \in \mathbb{N}}$ and the upper numbering filtration $\left(G^{r}\right)_{r \in \mathbb{Q},>0}$. In the classical case where the residue field is perfect, they are the same up to renumbering by the Herbrand function [40, Chapitre IV Section 3]. However, their properties make good contrasts. The lower one has an elementary definition and is compatible with subgroups while the upper one has more sophisticated definition and is compatible with quotients. The lower one is simply defined by $G_{i}=\operatorname{Ker}\left(G \rightarrow \operatorname{Aut}\left(\mathcal{O}_{L} / \mathfrak{m}_{L}^{i}\right)\right)$. More geometrically, it is rephrased as follows.

Take a presentation $\mathcal{O}_{K}\left[X_{1}, \ldots, X_{n}\right] /\left(f_{1}, \ldots, f_{n}\right) \rightarrow \mathcal{O}_{L}$ of the integer ring of $L$. We consider the $n$-dimensional closed disk $D^{n}$ defined by $\|x\| \leq 1$ over $K$ in the sense of rigid geometry and the morphism of disks $f: D^{n} \rightarrow D^{n}$ defined by $f_{1}, \ldots, f_{n}$. Then the Galois group $G$ is identified with the inverse image $f^{-1}(0)$ of the origin $0 \in D^{n}$. In other words, we have a cartesian diagram

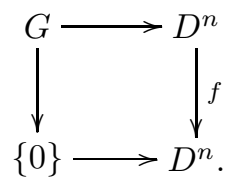

The subgroups $G_{i}$ and $G^{r}$ are defined to consist of the points of $G$ that are close to the identity in certain senses. For the lower one, the closeness is simply measured by the distance. Namely, the lower numbering subgroup $G_{i} \subset G$ consists of the points $\sigma \in G$ satisfying $d(\sigma$, id $) \leq\left|\pi_{L}^{i}\right|$ for a prime element $\pi_{L}$ of $L$.

To define the upper numbering filtration, we consider, for a rational number $r>0$, the inverse image $V_{r}=\left\{\left.x \in D^{n}|d(f(x), 0) \leq| \pi_{K}\right|^{r}\right\} \subset D^{n}$ of the 
closed subdisk of radius $\left|\pi_{K}\right|^{r}$, as an affinoid subdomain containing $G$. The upper numbering subgroup $G^{r}$ consists of the points in $G$ contained in the same geometric connected component of $V_{r}$ as the identity.

Theorem 2.1 ([4, Theorems 3.3, 3.8]). Let L be a finite Galois extension over K of Galois group $G=\operatorname{Gal}(L / K)$.

1. For a rational number $r>0$, the subset $G^{r} \subset G$ defined above is independent of the choice of presentation $\mathcal{O}_{K}\left[X_{1}, \ldots, X_{n}\right] /\left(f_{1}, \ldots, f_{n}\right) \rightarrow \mathcal{O}_{L}$ and is a normal subgroup of $G$. Further the inclusion $G \rightarrow V_{r}$ induces a bijection $G / G^{r} \rightarrow \pi_{0}\left(V_{r}\right)$ to the set of geometric connected components.

2. There exist rational numbers $0=r_{0}<r_{1}<\ldots<r_{m}$ such that $G^{r}=G^{r_{i}}$ for $r \in\left(r_{i-1}, r_{i}\right] \cap \mathbb{Q}$ and $i=1, \ldots, m$ and $G^{r}=1$ for $r>r_{m}$.

3. For a subfield $M \subset L$ Galois over $K$ and for a rational number $r>0$, the subgroup $\operatorname{Gal}(M / K)^{r} \subset \operatorname{Gal}(M / K)$ is the image of $G^{r}=\operatorname{Gal}(L / K)^{r}$.

The definition of the upper numbering filtration in the general residue field case is first found using rigid geometry, as described above. The use of rigid geometry is quite essential. For example, the proof of 2. in Theorem 2.1 relies on the reduced fiber theorem in rigid geometry [12]. However, an alternative scheme theoretic approach described below turned out to be quite powerful as well.

In the following, we give a definition of a logarithmic variant of the upper numbering filtration, that seems more essential than the non-logarithmic one and is defined using the natural log structure of the integer rings. For the generality on $\log$ schemes, we refer to [26] and [30, Section 4]. In the classical case where the residue field is perfect, the two filtrations are the same up to the shift by 1 . In the general residue field case, there is no simple relation among them. We emphasize here that both filtrations have scheme theoretic descriptions.

We regard $S=\operatorname{Spec} \mathcal{O}_{K}$ as a $\log$ scheme with the canonical log structure defined by the closed point $D_{S}=$ Spec $F$. Let $Q$ be a regular flat separated scheme of finite type over $S$. Assume that the reduced closed fiber $D_{Q}=\left(Q \times{ }_{S} D_{S}\right)_{\text {red }}$ is regular and that the $\log$ scheme $Q$ endowed with the $\log$ structure defined by $D_{Q}$ is log smooth over $S$. For example, $Q=\operatorname{Spec} \mathcal{O}_{K}\left[T_{1}, \ldots, T_{d}, U^{ \pm 1}\right] /\left(\pi-U T_{1}^{m}\right)$ for integers $d, m \geq 1$ and a prime element $\pi$ of $\mathcal{O}_{K}$.

Let $L$ be a finite Galois extension of $K$. We put $T=\operatorname{Spec} \mathcal{O}_{L}$ and $D_{T}=$ $\left(T \times{ }_{S} D_{S}\right)_{\text {red }}$. We consider a closed immersion $i: T \rightarrow Q$ that is exact in the sense that $D_{T}=D_{Q} \times{ }_{Q} T$. Let $P$ be a separated smooth scheme of finite type over $S$, $s: S \rightarrow P$ be a section and $f: Q \rightarrow P$ be a finite and flat morphism over $S$ such that the diagram

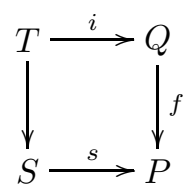

is cartesian. This diagram should be regarded as a scheme theoretic counterpart of (16). 
We consider a finite separable extension $K^{\prime}$ of $K$ containing $L$ as a subextension, in order to make a base change. We put $S^{\prime}=\operatorname{Spec} \mathcal{O}_{K^{\prime}}, F^{\prime}=\mathcal{O}_{K^{\prime}} / \mathfrak{m}_{K^{\prime}}$ and let $e=e_{K^{\prime} / K}$ be the ramification index.

Let $r>0$ be a rational number and assume that $r^{\prime}=e^{\prime} r$ is an integer. We regard the divisor $R^{\prime}=r^{\prime} D_{S^{\prime}}=\operatorname{Spec} \mathcal{O}_{K^{\prime}} / \mathfrak{m}_{K^{\prime}}^{r^{\prime}}$ of $S^{\prime}$ as a closed subscheme of $P_{S^{\prime}}=P \times_{S} S^{\prime}$ by the section $s^{\prime}: S^{\prime} \rightarrow P_{S^{\prime}}$ induced by $s: S \rightarrow P$. We consider the blow-up of $P_{S^{\prime}}$ at the center $R^{\prime}$ and let $P_{S^{\prime}}^{(r)}$ denote the complement of the proper transform of the closed fiber $P_{S^{\prime}} \times{ }_{S^{\prime}} D_{S^{\prime}}$. The scheme $P_{S^{\prime}}^{(r)}$ is smooth over $S^{\prime}$ and the closed fiber $P_{S^{\prime}}^{(r)} \times_{S^{\prime}} D_{S^{\prime}}$ is the vector bundle $\Theta_{F^{\prime}}^{(r)}$ over $F^{\prime}$ such that the set of $F^{\prime}$-valued points is the $F^{\prime}$-vector space $H_{o m} m_{F^{\prime}}\left(\mathfrak{m}_{K^{\prime}}^{r^{\prime}} / \mathfrak{m}_{K^{\prime}}^{r^{\prime}+1}, \mathcal{I}_{s(S)} / \mathcal{I}_{s(S)}^{2} \otimes_{\mathcal{O}_{K}} F^{\prime}\right)$ where $\mathcal{I}_{s(S)} \subset \mathcal{O}_{P_{S}}$ denotes the ideal sheaf.

We consider the normalizations $\bar{Q}_{S^{\prime}}^{(r)}$ and $\bar{T}_{S^{\prime}}$ of $Q \times{ }_{P} P_{S^{\prime}}^{(r)}$ and of $T \times{ }_{S} S^{\prime}$ respectively. Then, the diagram (17) induces a diagram

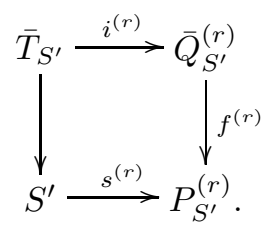

By the assumption that $K^{\prime}$ contains $L$, the scheme $\bar{T}_{S^{\prime}}$ is isomorphic to the disjoint union of finitely many copies of $S^{\prime}$ and the geometric fiber $\bar{T}_{\bar{F}}=\bar{T}_{S^{\prime}} \times_{S^{\prime}} \bar{F}$ is identified with $\operatorname{Gal}(L / K)$.

By Epp's theorem [17], after replacing $K^{\prime}$ by some finite separable extension, the geometric closed fiber $\bar{Q}_{\bar{F}}^{(r)}=\bar{Q}_{S^{\prime}}^{(r)} \times_{S^{\prime}}$ Spec $\bar{F}$ is reduced and the formation of $\bar{Q}_{S^{\prime}}^{(r)}$ commutes with further base change. We call such $\bar{Q}_{S^{\prime}}^{(r)}$ a stable integral model. The finite map $i^{(r)}: \bar{T}_{S^{\prime}} \rightarrow \bar{Q}_{S^{\prime}}^{(r)}$ induces surjections

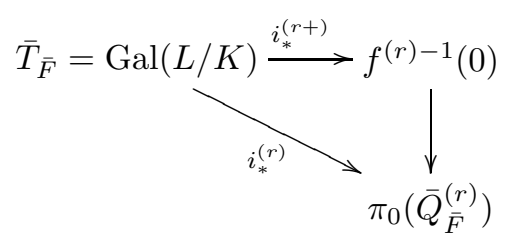

to the set of geometric connected components and to the inverse image of the origin $0 \in P_{\bar{F}}^{(r)}=\Theta_{\bar{F}}^{(r)}$.

Theorem 2.2 ([4, Theorems 3.11, 3.16], [38, Section 1.3]). Let L be a finite Galois extension over $K$ of Galois group $G$ and we consider a diagram (17) as above.

1. For a rational number $r>0$, we take a finite separable extension $K^{\prime}$ of $K$ containing $L$ such that $e_{K^{\prime} / K} r$ is an integer and that $Q_{\bar{S}^{\prime}}^{(r)}$ is a stable integral model.

Then, the inverse image $i_{*}^{(r)-1}\left(i_{*}^{(r)}(1)\right)=G_{\log }^{r} \subset G$ is independent of the choice of diagram (17) or an extension $K^{\prime}$ and is a normal subgroup of $G$. Further the surjection $i_{*}^{(r)}(19)$ induces a bijection $G / G_{\log }^{r} \rightarrow \pi_{0}\left(\bar{Q}_{\bar{F}}^{(r)}\right)$. 
2. Let the notation be as in 1 . Then, there exist rational numbers $0=r_{0}<$ $r_{1}<\ldots<r_{m}$ such that $G_{\log }^{r}=G_{\log }^{r_{i}}$ for $r \in\left(r_{i-1}, r_{i}\right] \cap \mathbb{Q}$ and $i=1, \ldots, m$ and $G_{\mathrm{log}}^{r}=1$ for $r>r_{m}$.

3. For a subfield $M \subset L$ Galois over $K$ and for a rational number $r>0$, the subgroup $\operatorname{Gal}(M / K)_{\log }^{r} \subset \operatorname{Gal}(M / K)$ is the image of $G^{r}=\operatorname{Gal}(L / K)_{\log }^{r}$.

For a rational number $r \geq 0$, we put $G_{\log }^{r+}=\bigcup_{s>r} G_{\log }^{s}$. Then, under the same assumption as in Theorem 2.2.1., the surjection $i_{*}^{(r+)}(19)$ induces a bijection $G / G_{\log }^{r+} \rightarrow f^{(r)-1}(0)$.

Example 2.1 ([25], [7]). If $K$ is of characteristic $p>0$, a cyclic extension $L$ of degree $p^{m+1}$ is defined by a Witt vector by the isomorphism $W_{m+1}(K) /(F-$ $1) \rightarrow H^{1}\left(K, \mathbb{Z} / p^{m+1} \mathbb{Z}\right)$ of Artin-Schreier-Witt theory. An increasing filtration on $W_{m+1}(K)$ is defined in [13] by

$F^{n} W_{m+1}(K)=\left\{\left(a_{0}, \ldots, a_{m}\right) \in W_{m+1}(K) \mid p^{m-i} v_{K}\left(a_{i}\right) \geq-n\right.$ for $\left.i=0, \ldots, m\right\}$.

The filtration induced by the surjection $W_{m+1}(K) \rightarrow H^{1}\left(K, \mathbb{Z} / p^{m+1} \mathbb{Z}\right)$ is considered in [25]. For $G=\operatorname{Gal}(L / K)$, the filtration $\left(G_{\log }^{n}\right)_{n \geq 0}$ indexed by integers is the dual of the restriction to $\operatorname{Hom}\left(\operatorname{Gal}(L / K), \mathbb{Z} / p^{m+1} \mathbb{Z}\right) \subset H^{1}\left(K, \mathbb{Z} / p^{m+1} \mathbb{Z}\right)$. Namely, we have $G_{\log }^{n}=\left\{\sigma \in G \mid c(\sigma)=0\right.$ if $\left.c \in F^{n} H^{1}\left(K, \mathbb{Z} / p^{m+1} \mathbb{Z}\right)\right\}$. Further, for a rational number $r \in(n-1, n] \cap \mathbb{Q}$, we have $G_{\log }^{r}=G_{\log }^{n}$.

Problem 2. Prove that, for an abelian extension in the mixed characteristic case, the filtration $\left(G_{\log }^{n}\right)_{n \geq 0}$ is the same as that defined by Kato in [25] and show $G_{\log }^{r}=G_{\log }^{n}$ for $r \in(n-1, n] \cap \mathbb{Q}$.

Definition 2.3. Let $L$ be a finite etale $K$-algebra and $r>0$ (resp. $r \geq 0$ ) be a rational number. Let $M$ be a finite Galois extension of $K$ such that $L \otimes_{K} M$ is isomorphic to the product of copies of $M$. Then, we say that the log ramification of $L$ over $K$ is bounded by $r$ (resp. by $r \geq 0)$ if the action of the subgroup $\operatorname{Gal}(M / K)_{\log }^{r}$ (resp. $\left.\operatorname{Gal}(M / K)_{\log }^{r+}\right)$ on the finite set $\operatorname{Hom}_{K}(L, M)$ is trivial.

It is interpreted geometrically as follows.

Proposition 2.4 ([38, Lemma 1.13]). Let $r>0$ be a rational number and we consider a diagram (17) and a finite separable extension $K^{\prime}$ of $K$ as in Theorem 2.2 such that $\bar{Q}_{S^{\prime}}^{(r)}$ is a stable integral model.

1. The following conditions are equivalent:

(1) The log ramification of $L$ over $K$ is bounded by $r$.

(2) The finite covering $\bar{Q}_{F^{\prime}}^{(r)} \rightarrow P_{F^{\prime}}^{(r)}$ is a split etale covering.

2. The following conditions are equivalent:

(1) The log ramification of $L$ over $K$ is bounded by $r+$.

(2) The finite map $\bar{Q}_{S^{\prime}}^{(r)} \rightarrow P_{S^{\prime}}^{(r)}$ is etale on a neighborhood of the closed fiber $\bar{Q}_{F^{\prime}}^{(r)}$. 
2.2. Ramification along a divisor. Let $X$ be a smooth separated scheme of finite type over a perfect field $k$ of characteristic $p>0$ and $U=X \backslash D$ be the complement of a divisor $D$ with simple normal crossings. We consider a finite etale $G$-torsor $V$ over $U$ for a finite group $G$ and study the ramification of $V$ along $D$. The ramification of $V$ along $D$ will be measured by linear combinations $R=\sum_{i} r_{i} D_{i}$ with rational coefficients $r_{i} \geq 0$ of irreducible components of $D$ as follows.

We consider the $\log$ product $P=X *_{k} X \subset\left(X \times_{k} X\right)^{\prime}$ and the log diagonal $\tilde{\delta}: X \rightarrow P=X *_{k} X$ as in Section 1.1. We define a relatively affine scheme $P^{(R)}$ over $P$. If the coefficients of $R=\sum_{i} r_{i} D_{i}$ are integers, the scheme $P^{(R)}$ is the complement of the proper transforms of $P \times_{X} R$ in the blow-up of $P$ at the center $R \subset X$ embedded by the log diagonal map $\tilde{\delta}: X \rightarrow P$. In other words, $P^{(R)}$ is the relatively affine scheme over $P$ defined by the quasi-coherent $\mathcal{O}_{P^{-}}$ algebra $\mathcal{O}_{P}\left[\mathcal{I}_{X}\left(R_{P}\right)\right]=\sum_{n>0} \mathcal{I}_{X}^{n}\left(n R_{P}\right)$ where $\mathcal{I}_{X} \subset \mathcal{O}_{P}$ is the ideal sheaf defining the $\log$ diagonal and the divisor $R_{P} \subset P$ is the pull-back of $R \subset X$. The base change $P^{(R)} \times_{X} R$ with respect to the projection $P^{(R)} \rightarrow X \supset R$ is the twisted tangent bundle $\Theta^{(R)}=\mathbf{V}\left(\Omega_{X}^{1}(\log D)(R)\right) \times_{X} R$ where $\mathbf{V}\left(\Omega_{X}^{1}(\log D)(R)\right)$ denotes the vector bundle defined by the symmetric algebra of the locally free $\mathcal{O}_{X}$-module $\Omega_{X}^{1}(\log D)(R)$.

For general $R$, it is defined by the quasi-coherent $\mathcal{O}_{P}$-algebra $\sum_{n \geq 0} \mathcal{I}_{X}^{n}\left(\left\lfloor n R_{P}\right\rfloor\right)$ where $\left\lfloor n R_{P}\right\rfloor$ denotes the integral part. The log diagonal $\tilde{\delta}: X \rightarrow P=X *_{k} X$ is uniquely lifted to an immersion $\delta^{(R)}: X \rightarrow P^{(R)}$. The open immersion $U \times_{k} U \rightarrow$ $X *_{k} X=P$ is lifted to an open immersion $j^{(R)}: U \times_{k} U \rightarrow P^{(R)}$.

Example 2.2. Assume $X=\operatorname{Spec} k\left[T_{1}, \ldots, T_{d}\right]$ and $D$ is defined by $T_{1} \cdots T_{n}$ for $0 \leq n \leq d$. Then, the $\log$ product $P=X *_{k} X$ is the spectrum of

$$
A=k\left[T_{1}, \ldots, T_{d}, S_{1}, \ldots, S_{d}, U_{1}^{ \pm 1}, \ldots, U_{n}^{ \pm 1}\right] /\left(S_{1}-U_{1} T_{1}, \ldots, S_{n}-U_{n} T_{n}\right)
$$

and the $\log$ diagonal $\tilde{\delta}: X \rightarrow P=X *_{k} X$ is defined by $U_{1}=\cdots=U_{n}=1$ and $T_{n+1}=S_{n+1}, \ldots, T_{d}=S_{d}$.

Further assume that the coefficients of $R=\sum_{i=1}^{n} r_{i} D_{i}$ are integral. Then, if we put $T^{R}=T_{1}^{r_{1}} \cdots T_{n}^{r_{n}}$, the scheme $\left(X *_{k} X\right)^{(R)}$ is the spectrum of

$$
\begin{aligned}
& A\left[V_{1}, \ldots, V_{d}\right] /\left(U_{1}-1-V_{1} T^{R}, \ldots, U_{n}-1-V_{n} T^{R},\right. \\
& \left.S_{n+1}-T_{n+1}-V_{n+1} T^{R}, \ldots, S_{d}-T_{d}-V_{d} T^{R}\right) \\
= & k\left[T_{1}, \ldots, T_{d}, V_{1}, \ldots, V_{d},\left(1+V_{1} T^{R}\right)^{-1}, \ldots,\left(1+V_{n} T^{R}\right)^{-1}\right] .
\end{aligned}
$$

The immersion $\delta^{(R)}: X \rightarrow\left(X *_{k} X\right)^{(R)}$ is defined by $V_{1}=\cdots=V_{d}=0$.

Let $V$ be a $G$-torsor over $U$ for a finite group $G$. We consider the quotient $\left(V \times \times_{k} V\right) / \Delta G$ by the diagonal $\Delta G \subset G \times G$ as a finite etale covering of $U \times_{k} U$ and let $Z$ be the normalization of $\left(X *_{k} X\right)^{(R)}$ in the quotient $\left(V \times_{k} V\right) / \Delta G$. The diagonal map $V \rightarrow V \times_{k} V$ induces a closed immersion $U=V / G \rightarrow\left(V \times_{k} V\right) / \Delta G$ on the quotients and is extended to a closed immersion $e: X \rightarrow Z$. 
Definition 2.5. Let $V$ be a $G$-torsor over $U$ for a finite group $G$. We say that the ramification of $V$ over $U$ is bounded by $R+$ if the normalization $Z$ of $\left(X *_{k} X\right)^{(R)}$ in the quotient $\left(V \times_{k} V\right) / \Delta G$ is etale over $\left(X *_{k} X\right)^{(R)}$ on a neighborhood of the image of $e: X \rightarrow Z$.

The following is an immediate consequence of Proposition 2.4.2.

Lemma 2.6. Assume $D$ is irreducible and let $K=$ Frac $\widehat{\mathcal{O}}_{X, \xi}$ be the fraction field of the completion of the local ring $\mathcal{O}_{X, \xi}$ at the generic point $\xi$ of $D$. Then, for a rational number $r \geq 0$, the following conditions are equivalent:

(1) The log ramification of the etale $K$-algebra $\Gamma\left(V \times_{U} \operatorname{Spec} K, \mathcal{O}\right)$ is bounded by $r+$.

(2) There exists an open neighborhood $X^{\prime}$ of $\xi$ such that the ramification of $V \cap X^{\prime}$ over $U \cap X^{\prime}$ is bounded by $r\left(D \cap X^{\prime}\right)+$.

By the following lemma, the general case is reduced to the case where the coefficients of $R$ are integral.

Lemma 2.7. Let $f: X^{\prime} \rightarrow X$ be a morphism of separated smooth schemes of finite type over $k$. Let $U \subset X$ and $U^{\prime} \subset X^{\prime}$ be the complements of divisors with simple normal crossings respectively satisfying $U^{\prime} \subset f^{-1}(U)$.

Let $V \rightarrow U$ be a $G$-torsor for a finite group $G$ and $V^{\prime}=V \times_{U} U^{\prime} \rightarrow U^{\prime}$ be the pull-back. Let $R=\sum_{i} r_{i} D_{i} \geq 0$ be an effective divisor with rational coefficients and $R^{\prime}=f^{*} R$ be the pull-back. We consider the following conditions.

(1) The ramification of $V$ is bounded by $R+$.

(2) The ramification of $V^{\prime}$ is bounded by $R^{\prime}+$.

We always have an implication $(1) \Rightarrow(2)$. Conversely, if $f: X^{\prime} \rightarrow X$ is log smooth and is faithfully flat and if $U^{\prime}=f^{-1}(U)$, we have the other implication $(2) \Rightarrow(1)$.

The main result is the following.

Theorem 2.8. Let $X$ be a separated smooth scheme of finite type over $k$ and $U=X \backslash D$ be the complement of a divisor with simple normal crossings. Assume that the coefficients of $R=\sum_{i} r_{i} D_{i} \geq 0$ are integral. Let $V$ be a $G$-torsor over $U$ for a finite group $G$ and $Z_{0} \subset Z$ be the maximum open subscheme etale over $\left(X *_{k} X\right)^{(R)}$ of the normalization $Z$ of $\left(V \times_{k} V\right) / \Delta G$. Let $e: X \rightarrow Z$ be the section induced by the diagonal.

Then, the base change $Z_{0, R}=Z_{0} \times_{X} R$ with respect to the projection $Z_{0} \rightarrow$ $\left(X *_{k} X\right)^{(R)} \rightarrow X \supset R$ has a natural structure of smooth commutative group scheme over $R$ such that the map $e_{R}: X_{R} \rightarrow Z_{0, R}$ induced by $e: X \rightarrow Z$ is the unit. Further the etale map $Z_{0, R} \rightarrow \Theta^{(R)}=\left(X *_{k} X\right)^{(R)} \times_{X} R$ induced by the canonical map $Z \rightarrow\left(X *_{k} X\right)^{(R)}$ is a group homomorphism.

For every point $x \in R$, the connected component $Z_{0, x}^{0}$ of the fiber $Z_{0, x}$ is isomorphic to the product of finitely many copies of the additive group $\mathbf{G}_{a, x}$ and the $\operatorname{map} Z_{0, x}^{0} \rightarrow \Theta_{x}^{(R)}$ is an etale isogeny. 
Problem 3. Prove an analogous result for schemes over a discrete valuation rings with perfect residue field.

Theorem 2.8 has the following application to the filtration by ramification groups in the equal characteristic case.

Let $K$ be a complete discrete valuation field of characteristic $p>0$ and assume that the residue field $F$ has a finite $p$-basis. Let $\Omega_{\mathcal{O}_{K}}^{1}(\log )$ denote the $\mathcal{O}_{K^{-}}$ submodule of the $K$-vector space $\Omega_{K}^{1}$ generated by $\Omega_{\mathcal{O}_{K}}^{1}$ and $d \log \pi$ for a prime element $\pi$ of $K$. By abuse of notation, let $\Omega_{F}^{1}(\log )$ denote the $F$-vector space $\Omega_{\mathcal{O}_{K}}^{1}(\log ) \otimes_{\mathcal{O}_{K}} F$. Then, we have an exact sequence $0 \rightarrow \Omega_{F}^{1} \rightarrow \Omega_{F}^{1}(\log ) \stackrel{\text { res }}{\rightarrow} F \rightarrow 0$ of $F$-vector spaces of finite dimension. We extend the normalized discrete valuation $v$ of $K$ to a separable closure $\bar{K}$ and, for a rational number $r$, we put $\mathfrak{m}_{\bar{K}}^{r}=\{a \in \bar{K} \mid v(a) \geq r\}$ and $\mathfrak{m}_{\bar{K}}^{r+}=\{a \in \bar{K} \mid v(a)>r\}$. The $\bar{F}$-vector space $\mathfrak{m}_{\bar{K}}^{r} / \mathfrak{m}_{\bar{K}}^{r+}$ is of dimension 1 .

Corollary ([5, Theorem 2.15], [38, Theorem 1.24, Corollary 1.25]). Let $K$ be a complete discrete valuation field of characteristic $p>0$ and $L$ be a finite Galois extension of Galois group $G$. Then, for a rational number $r>0$, the graded quotient $\mathrm{Gr}_{\log }^{r} G=G_{\log }^{r} / G_{\log }^{r+}$ is abelian and killed by $p$.

If $F$ has a finite $p$-basis, there exists a canonical injection

$$
\operatorname{Hom}\left(\operatorname{Gr}_{\log }^{r} G, \mathbb{F}_{p}\right) \rightarrow \operatorname{Hom}_{\bar{F}}\left(\mathfrak{m}_{\bar{K}}^{r} / \mathfrak{m}_{\bar{K}}^{r+}, \Omega_{F}^{1}(\log ) \otimes_{F} \bar{F}\right) .
$$

For a non-trivial character $\chi \in \operatorname{Hom}\left(\operatorname{Gr}_{\log }^{r} G, \mathbb{F}_{p}\right)$, we call the image rsw $\chi \in$ $\operatorname{Hom}_{\bar{F}}\left(\mathfrak{m}_{\bar{K}}^{r} / \mathfrak{m}_{\bar{K}}^{r+}, \Omega_{F}^{1}(\log ) \otimes_{F} \bar{F}\right)$ the refined Swan character of $\chi$.

For $K$ of mixed characteristic, one has an analogous result. The proof is similar but technically more difficult.

Problem 4. Determine the union of the images of the injections (22) for all finite Galois extensions $L \subset \bar{K}$ over $K$.

In the classical case where the residue field is perfect, the union is the whole.

Example 2.3 ([25], [7]). We keep the notation in Example 2.1. We define a canonical map $F^{m} d: W_{m+1}(K) \rightarrow \Omega_{K}^{1}$ by sending $\left(a_{0}, \ldots, a_{m}\right)$ to $a_{0}^{p^{m}-1} d a_{0}+$ $\cdots+d a_{m}$. It maps $F^{n} W_{m+1}(K)$ to $F^{n} \Omega_{K}^{1}=\mathfrak{m}_{K}^{-n} \Omega_{\mathcal{O}_{K}}^{1}(\log )$ for $n \in \mathbb{Z}$ and induces an injection

$$
\operatorname{Gr}^{n} H^{1}\left(K, \mathbb{Z} / p^{m+1} \mathbb{Z}\right) \rightarrow \operatorname{Gr}^{n} \Omega_{K}^{1}=\operatorname{Hom}_{F}\left(\mathfrak{m}_{K}^{n} / \mathfrak{m}_{K}^{n+1}, \Omega_{F}^{1}(\log )\right)
$$

for $n>0$.

Let $L$ be a cyclic extension of degree $p^{m+1}$ corresponding to a character $\chi \in$ $H^{1}\left(K, \mathbb{Z} / p^{m+1} \mathbb{Z}\right)$. The smallest integer $n \geq 0$ such that $\chi \in F^{n} H^{1}\left(K, \mathbb{Z} / p^{m+1} \mathbb{Z}\right)$ is called the conductor of $\chi$ and is equal to the smallest rational number $r$ such that the ramification of $L$ is bounded by $r+$. The character is ramified if and only if the conductor is $>0$. For a ramified character $\chi$ of conductor $n>0$, the image of the class of $\chi$ by the injection (23) in $\operatorname{Hom}_{F}\left(\mathfrak{m}_{K}^{n} / \mathfrak{m}_{K}^{n+1}, \Omega_{F}^{1}(\log )\right) \subset$ $\operatorname{Hom}_{\bar{F}}\left(\mathfrak{m}_{\bar{K}}^{n} / \mathfrak{m}_{\bar{K}}^{n+}, \Omega_{F}^{1}(\log ) \otimes_{F} \bar{F}\right)$ is the refined Swan character rsw $\chi$. 
2.3. Characteristic cycles. We keep the notation in Section 2.2 that $X$ is a separated smooth scheme of dimension $d$ over a perfect field $k$ of characteristic $p>0$ and $U=X \backslash D$ is the complement of a divisor with simple normal crossings. For each irreducible component $D_{i}$ of $D$, let $\xi_{i}$ be the generic point of $D_{i}$ and $K_{i}=$ Frac $\widehat{\mathcal{O}}_{X, \xi_{i}}$ be the local field. Recall that, for a divisor $R=\sum_{i} r_{i} D_{i}$ with rational coefficients $r_{i} \geq 0$, we have a cartesian diagram

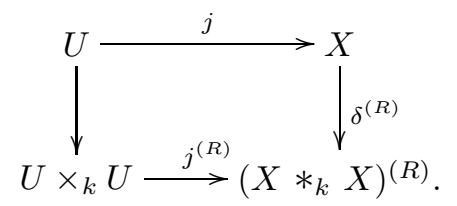

For a smooth sheaf on $U$, we make a definition similar to Definition 2.5. As a coefficient ring $\Lambda$, we consider a ring finite over $\mathbb{Z} / \ell^{n} \mathbb{Z}, \mathbb{Z}_{\ell}$ or $\mathbb{Q}_{\ell}$ for a prime number $\ell \neq$ char $k$ as in Section 1.2.

Definition 2.9 ([38, Definition 2.19]). Let $\mathcal{F}$ be a smooth sheaf of $\Lambda$-modules on $U$ and we put $\mathcal{H}_{0}=\mathcal{H}$ om $\left(\operatorname{pr}_{2}^{*} \mathcal{F}, \operatorname{pr}_{1}^{*} \mathcal{F}\right)$ on $U \times_{k} U$. We say that the ramification of $\mathcal{F}$ is bounded by $R+$ if the identity $\operatorname{id}_{\mathcal{F}}$ is in the image of the base change map

$$
\Gamma\left(X, \delta^{(R) *} j_{*}^{(R)} \mathcal{H}_{0}\right) \rightarrow \Gamma\left(X, j_{*} \mathcal{E} n d(\mathcal{F})\right)=\operatorname{End}(\mathcal{F}) .
$$

The following is an immediate consequence of Definition.

Lemma 2.10. The following conditions are equivalent:

(1) The ramification of $\mathcal{F}$ is bounded by $R+$.

(2) The base change map $\delta^{(R) *} j_{*}^{(R)} \mathcal{H}_{0} \rightarrow j_{*} \mathcal{E} n d(\mathcal{F})$ is an isomorphism.

Example 2.4 ([6, Proposition 4.2.2]). Let $\mathcal{F}$ be a smooth sheaf of rank 1 corresponding to a character $\chi: \pi_{1}(U)^{\mathrm{ab}} \rightarrow \Lambda^{\times}$. For each irreducible component $D_{i}$, let $K_{i}$ be the local field and $n_{i}$ be the conductor of the $p$-part of the character $\chi_{i}: G_{K_{i}}^{\mathrm{ab}} \rightarrow \Lambda^{\times}$. We put $R=\sum_{i} n_{i} D_{i}$. Then, the ramification of $\mathcal{F}$ is bounded by $R+$. Further, $j_{*}^{(R)} \mathcal{H}_{0}$ is a smooth sheaf of $\Lambda$-modules of rank 1 on $\left(X *_{k} X\right)^{(R)}$. For a component with $r_{i}>0$, the restriction of $j_{*}^{(R)} \mathcal{H}_{0}$ to the fiber $\Theta_{\xi_{i}}^{(R)}$ is the Artin-Schreier sheaf defined by the refined Swan character rsw $\chi_{i}$ regarded as a linear form on $\Theta_{\xi_{i}}^{(R)}$.

In the remaining part of the article, we present a computation of the characteristic class $C(j ! \mathcal{F}) \in H^{2 d}(X, \Lambda(d))$ for a smooth $\ell$-adic sheaf $\mathcal{F}$ on $U$ whose ramification is bounded by $R+$ using the geometric ramification theory under a certain assumption. We assume that the coefficients of $R=\sum_{i} r_{i} D_{i}$ are integral for simplicity. For the general case, we refer to [38, Section 3].

For each irreducible component $D_{i}$ of $D$ such that $r_{i}>0$, we consider the $\ell$-adic representation $M_{i}$ of the local field $K_{i}=$ Frac $\widehat{\mathcal{O}}_{X, \xi_{i}}$ defined by $\mathcal{F}$. By the assumption that the ramification of $\mathcal{F}$ is bounded by $R+$, the subgroup $G_{K_{i} \text {, log }}^{r_{i}+}$ acts trivially on $M_{i}$. We assume the following condition: 
- The $G_{K_{i}, \log }^{r_{i}}$-fixed part of $M_{i}$ is 0 .

This condition means that, for each irreducible component, the wild ramification of $\mathcal{F}$ at the generic point is homogeneous. The restriction to $G_{K_{i}, \log }^{r_{i}}$ is then decomposed as the sum $\left.M_{i}\right|_{G_{K_{i}, \log }^{r_{i}}}=\bigoplus_{j} \chi_{i j}^{\oplus m_{i j}}$ of non-trivial characters of $\mathrm{Gr}_{\log }^{r_{i}} G_{K_{i}}$. By the assumption that $r_{i}$ is an integer, the refined Swan character defines a non-trivial $\bar{F}_{i}$-linear homomorphism

$$
\operatorname{rsw} \chi_{i j}: \mathfrak{m}_{K_{i}}^{r_{i}} / \mathfrak{m}_{K_{i}}^{r_{i}+1} \otimes_{F_{i}} \bar{F}_{i} \rightarrow \Omega_{X}^{1}(\log D)_{\xi_{i}} \otimes \bar{F}_{i},
$$

where $F_{i}=\kappa\left(\xi_{i}\right)$ is the function field of $D_{i}$. Let $E_{i j}$ be a finite extension of $F_{i}$ such that rsw $\chi_{i j}$ is defined and let $T_{i j}$ be the normalization of $D_{i}$ in $E_{i j}$.

We assume further the following condition:

(C) The refined Swan character rsw $\chi_{i j}$ defines a locally splitting injection

$$
\text { rsw } \chi_{i j}: \mathcal{O}_{X}(-R) \otimes_{\mathcal{O}_{X}} \mathcal{O}_{T_{i j}} \rightarrow \Omega_{X}^{1}(\log D) \otimes_{\mathcal{O}_{X}} \mathcal{O}_{T_{i j}} .
$$

This condition says that for each irreducible component, the wild ramification of $\mathcal{F}$ is controlled at the generic point. In the rank one case, the condition $(\mathrm{C})$ is called the cleanness condition and studied in [28]. The key ingredient in the proof of the following computation is Theorem 2.8.

Proposition 2.11 ([38, Corollary 3.3]). Assume the condition (C) above is satisfied. Let $\pi^{(R)}:\left(X *_{k} X\right)^{(R)} \rightarrow X \times_{k} X$ denote the canonical map. We put $\mathcal{H}_{0}=$ $\mathcal{H o m}\left(\operatorname{pr}_{2}^{*} \mathcal{F}, \operatorname{pr}_{1}^{*} \mathcal{F}\right)$ on $U \times_{k} U$ and $\mathcal{H}=R \mathcal{H}$ om $\left(\operatorname{pr}_{2}^{*} j_{!} \mathcal{F}, R \operatorname{pr}_{1}^{!} j_{!} \mathcal{F}\right)$ on $X \times_{k} X$. We regard the identity $\mathrm{id}_{\mathcal{F}}$ as an element of $H_{X}^{0}\left(X \times_{k} X, \mathcal{H}\right)$ and of $H^{0}\left(X, \delta^{(R) *} j_{*}^{(R)} \mathcal{H}_{0}\right)$ by the isomorphisms $\operatorname{End}(j ! \mathcal{F}) \rightarrow H_{X}^{0}\left(X \times_{k} X, \mathcal{H}\right)(6)$ and $H^{0}\left(X, \delta^{(R) *} j_{*}^{(R)} \mathcal{H}_{0}\right) \rightarrow$ $\operatorname{End}(\mathcal{F})(24)$.

Then, the image of the identity $\operatorname{id}_{\mathcal{F}}$ by the pull-back map

$$
H_{X}^{0}\left(X \times_{k} X, \mathcal{H}\right) \longrightarrow H_{\pi^{(R)-1}(X)}^{2 d}\left(\left(X *_{k} X\right)^{(R)}, j_{*}^{(R)} \mathcal{H}_{0}(d)\right)
$$

is equal to the image of the cup-product $[X] \cup \mathrm{id}_{\mathcal{F}} \in H_{X}^{2 d}\left(\left(X *_{k} X\right)^{(R)}, j_{*}^{(R)} \mathcal{H}_{0}(d)\right)$ of the cycle class $[X] \in H_{X}^{2 d}\left(\left(X *_{k} X\right)^{(R)}, \Lambda(d)\right)$ with $\operatorname{id}_{\mathcal{F}} \in H^{0}\left(X, \delta^{(R) *} j_{*}^{(R)} \mathcal{H}_{0}\right)$.

Corollary ([38, Theorem 3.4]). For the characteristic class, we have an equality

$$
C(j ! \mathcal{F})=\operatorname{rank} \mathcal{F} \cdot(X, X)_{\left(X *_{k} X\right)^{(R)}} .
$$

Consequently, if $X$ is proper, we have

$$
\chi_{c}\left(U_{\bar{k}}, \mathcal{F}\right)=\operatorname{rank} \mathcal{F} \times \operatorname{deg}(X, X)_{\left(X *_{k} X\right)^{(R)}} .
$$

We keep the assumptions and define the characteristic cycle in order to describe the computation in Corollary more geometric terms. We call the vector bundle over $X$ defined by the symmetric $\mathcal{O}_{X}$-algebra of the dual module $\Omega_{X}^{1}(\log D)^{*}$ the 
logarithmic cotangent bundle $T^{*} X(\log D)$. Let $L$ denote the line bundle over $X$ defined by the symmetric $\mathcal{O}_{X}$-algebra of $\mathcal{O}_{X}(R)$. By the condition $(\mathrm{C})$, the refined Swan character rsw $\chi_{i j}$ defines a linear map

$$
r_{i j}: L \times_{X} T_{i j} \rightarrow T^{*} X(\log D) \times_{X} T_{i j} .
$$

We define the characteristic cycle $C C(\mathcal{F})$ by

$$
C C(\mathcal{F})=(-1)^{d}\left(\operatorname{rank} \mathcal{F} \cdot[X]+\sum_{i} \sum_{j} \frac{m_{i j}}{\left[E_{i j}: F_{i}\right]} \operatorname{pr}_{1 *} r_{i j *}\left[L \times_{X} T_{i j}\right]\right)
$$

as a dimension $d$-cycle of $T^{*} X(\log D)$. In the first term of the right hand side, $[X]$ denotes the class of the 0 -section. In the second term, $\operatorname{pr}_{1 *} r_{i j *}\left[L \times_{X} T_{i j}\right]$ denote the image of the class $\left[L \times_{X} T_{i j}\right]$ by the composition $L \times_{X} T_{i j} \rightarrow T^{*} X(\log D) \times_{X} T_{i j} \rightarrow$ $T^{*} X(\log D)$. The reason why the characteristic cycle defined above is determined by points of codimension 1 is the condition $(\mathrm{C})$.

As a consequence of Corollary, we have the following.

Theorem 2.12 ([38, Theorem 3.7]). Assume the condition (C).

1. The characteristic class $C\left(j_{!} \mathcal{F}\right)$ is equal to the pull-back by the 0-section $0: X \rightarrow T^{*} X(\log D)$ of the cycle class of the characteristic cycle $C C(\mathcal{F})$ :

$$
C(j ! \mathcal{F})=0^{*}[C C(\mathcal{F})] .
$$

2. Assume further that $X$ is proper. Then the Euler number $\chi_{c}\left(U_{\bar{k}}, \mathcal{F}\right)$ is equal to the intersection number of the 0-section with the characteristic cycle:

$$
\chi_{c}\left(U_{\bar{k}}, \mathcal{F}\right)=(X, C C(\mathcal{F}))_{T^{*} X(\log D)} .
$$

Problem 5. Find an intrinsic definition of the characteristic cycle.

\section{References}

[1] A. Abbes, Cycles on arithmetic surfaces, Compositio Math. 122 (2000), no. 1, 23-111.

[2] — , The Grothendieck-Ogg-Shafarevich formula for arithmetic surfaces, J. Algebraic Geom. 9 (2000), no. 3, 529-576.

[3] A. Abbes, A. Mokrane, Sous-groupes canoniques et cycles évanescents p-adiques pour les variétés abéliennes, Publ. Math. IHES 101, (2004), 117-162

[4] A. Abbes, T. Saito, Ramification of local fields with imperfect residue fields, Amer. J. of Math. 124 (2002), 879-920

[5] —, Ramification of local fields with imperfect residue fields II, Documenta Math., Extra Volume K. Kato (2003), 3-70.

[6] - The characteristic class and ramification of an $\ell$-adic étale sheaf, Invent. Math., 168 (2007) 567-612. 
[7] _ - Analyse micro-locale $\ell$-adique en caractéristique $p>0$ : Le cas d'un trait, Publ. RIMS 45-1 (2009) 25-74.

[8] — Local Fourier transform and epsilon factors, arXiv:0809.0180 accepted for publication at Compo. Math.

[9] T. Abe, Comparison between Swan conductors and characteristic cycles, accepted for publication at Compo. Math.

[10] P. Berthelot, Introduction à la théorie arithmétique des $\mathcal{D}$-modules, Cohomologies p-adiques et applications arithmétiques, II. Astérisque 279 (2002), 1-80.

[11] S. Bloch, Cycles on arithmetic schemes and Euler characteristics of curves, Algebraic geometry, Bowdoin, 1985, 421-450, Proc. Sympos. Pure Math. 46, Part 2, Amer. Math. Soc., Providence, RI, 1987.

[12] S. Bosch, W. Lütkebohmert, and M. Raynaud, Formal and rigid geometry. IV. The reduced fibre theorem, Invent. Math. 119 (1995), 361-398.

[13] J.-L. Brylinski, Théorie du corps de classes de Kato et revêtements abéliens de surfaces, Ann. Inst. Fourier 33 (1983), 23-38.

[14] A. J. de Jong, Families of curves and alterations, Ann. Inst. Fourier (Grenoble) 47 (1997), no. 2, 599-621.

[15] P. Deligne, Équations différentielles à points singuliers réguliers, LNM 163, Springer-Verlag, Berlin-New York, 1970.

[16] — L Letter to Illusie, Nov. 4, 1976

[17] H. P. Epp, Eliminating wild ramification, Invent. Math. 19 (1973), 235-249.

[18] A. Grothendieck, rédigé par L. Illusie, Formule de Lefschetz, exposé III, SGA 5, Springer LNM 589 (1977) 73-137.

[19] A. Grothendieck, rédigé par I. Bucur, Formule d'Euler-Poincaré en cohomologie étale, exposé X, SGA 5, Springer LNM 589 (1977) 372-406.

[20] G. Faltings, Crystalline cohomology and p-adic Galois-representations, Algebraic analysis, geometry, and number theory (Baltimore, MD, 1988), 25-80, Johns Hopkins Univ. Press, Baltimore, MD, 1989.

[21] S. Hattori, Tame characters and ramification of finite flat group schemes, J. of Number theory 128 (2008), 1091-1108

[22] L. Illusie, Théorie de Brauer et caractéristique d'Euler-Poincaré, d'après P. Deligne, Astérisques 82-83, SMF, (1981), 161-172.

[23] V. Cossart, U. Jannsen and S. Saito, Canonical embedded and non-embedded resolution of singularities for excellent two-dimensional schemes, Preprint 2009, arXiv:math.AG/0905.2191

[24] M. Kashiwara, P. Schapira, Sheaves on manifolds, Springer-Verlag (1990).

[25] K. Kato, Swan conductors for characters of degree one in the imperfect residue field case, Algebraic K-theory and algebraic number theory (Honolulu, HI, 1987), 101-131, Contemp. Math., 83, Amer. Math. Soc., Providence, RI, 1989.

[26] — Logarithmic structures of Fontaine-Illusie, Algebraic analysis, geometry, and number theory, (J.-I. Igusa ed.), Johns Hopkins UP, Baltimore, 1989, 191-224.

[27] —, Generalized class field theory, Proceedings of ICM (Kyoto, 1990), 419-428, Math. Soc. Japan, Tokyo, 1991. 
[28] — Class field theory, D-modules, and ramification of higher dimensional schemes, Part I, American J. of Math., 116 (1994), 757-784.

[29] K. Kato, S. Saito, T. Saito, Artin characters for algebraic surfaces, American J. of Math. 109 (1987), 49-76.

[30] K. Kato, T. Saito, On the conductor formula of Bloch, Publ. Math. IHES 100, (2004), 5-151.

[31] — Ramification theory for varieties over a perfect field, Ann. of Math., 168 (2008), 33-96.

[32] G. Laumon, Caractéristique d'Euler-Poincaré des faisceaux constructibles sur une surface, Astérisques, 101-102 (1982), 193-207.

[33] X. Liang, Preprints on his webpage http://math.uchicago.edu/ lxiao/papers.htm

[34] A. P. Ogg, Elliptic curves and wild ramification, Amer. J. Math. 89 (1967) 1-21.

[35] F. Orgogozo, Conjecture de Bloch et nombres de Milnor, Annales de l'Institut Fourier 53, no. 6 (2003) 1739-1754

[36] R. Pink, On the calculation of local terms in the Lefschetz-Verdier trace formula and its application to a conjecture of Deligne, Ann. of Math. (2) 135 (1992), no. 3, $483-525$.

[37] Mme M. Raynaud (d'après notes inédites de A. Grothendieck), Propreté cohomologique des faisceaux d'ensembles et des faisceaux de groupes non commutatifs, exposé XIII, SGA 1, Springer LNM 224 (1971), Édition recomposée SMF (2003).

[38] T. Saito, Wild ramification and the characteristic cycle of an $\ell$-adic sheaf, Journal de l'Institut de Mathematiques de Jussieu, (2009) 8(4), 769-829

[39] J-P. Serre, Sur la rationalité des représentations d'Artin, Ann. of Math. 72 (1960), 406-420.

[40] — Corps Locaux, Hermann, Paris, France, 1962.

[41] —, Représentations linéaires des Groupes finis, Hermann, Paris, France, 1967.

[42] Y. Tian, Canonical subgroups of Barsotti-Tate groups, accepted for publication at Ann. of Math.

[43] T. Tsushima, On localization of the characteristic class of $\ell$-adic sheaves and relative Kato-Saito conductor formula in characteristic $p>0$, preprint.

[44] I. Vidal, Théorie de Brauer et conducteur de Swan, J. Algebraic Geom. 13 (2004), 349-391.

Department of Mathematical Sciences, University of Tokyo,

Tokyo, 153-8914, Japan

E-mail: t-saito@ms.u-tokyo.ac.jp 